\title{
THE UNUSUAL SHORT PERIOD BINARY V342 AQUILAE; DOES IT BREAK THE EVOLUTIONARY THEORY MOLD?
}

\author{
C. N. HARTMAN \\ The Catholic University of America and Goddard Space Flight Center \\ chartman@iue.gsfc.nasa.gov \\ R. S. POLIDAN \\ Goddard Space Flight Center \\ A. D. WELTY \\ Space Telescope Science Institute \\ R. WADE \\ Pennsylvania State University \\ P. B. ETZEL \\ San Diego State University \\ AND \\ F. C. BRUHWEILER \\ The Catholic University of America
}

Analysis of the eclipsing (period=3.39 days) binary V342 Aquilae at visible wavelengths reveals that the system is heavily enshrouded with gas, masking the identity of the underlying stars. In the ultraviolet V342 Aquilae shows six times more attenuation at phases outside of eclipse than most Algols. It is likely, then, that V342 Aquilae is the most enshrouded short period Algol system known. Our ultraviolet spectra show very pronounced Fe II absorption lines arising from ground and metastable levels, indicating an extensive circumstellar gas envelope. The strength of this absorption shows both an orbital and a cycle-to-cycle variability. The eclipse spectra display very strong emission from lines such as C II, Si IV, and C IV, with a striking similarity to the interacting binary TT Hydrae (B9.5V+K0III). We have deduced that the system consists of a late B star with a late GIII-IV companion and a mass ratio on the order of 0.5 . A detailed description of the observations, as well as deduced geometrical and physical properties of the system are presented. The apparent conflict of these observations with presently understood theory of binary evolution will be discussed. 